\title{
Established Serum Biomarkers Are Prognostic Factors in Patients With Oligometastatic Cancer and Brain Involvement
}

\author{
CARSTEN NIEDER $^{1,2}$, ASTRID DALHAUG $^{1}$ and BÅRD MANNSÅKER ${ }^{1}$ \\ ${ }^{1}$ Department of Oncology and Palliative Medicine, Nordland Hospital, Bod $\phi$, Norway; \\ ${ }^{2}$ Department of Clinical Medicine, Faculty of Health Sciences, \\ UiT - The Arctic University of Norway, Troms $\phi$, Norway
}

\begin{abstract}
Background/Aim: This study was designed to evaluate the prognostic impact of the previously validated LabBM score (serum lactate dehydrogenase, C-reactive protein, albumin, hemoglobin, platelets) in a new setting, namely patients with a limited number of brain metastases, arbitrarily defined as max. 4 brain lesions, from common tumor types such as lung and breast cancer. A total of 5 metastatic lesions overall were allowed to comply with current definitions of oligometastatic cancer. Patients and Methods: For this retrospective single-institution analysis, 101 patients were identified from a previously described, prospectively maintained database. Results: Twenty-one patients $(21 \%)$ had extracranial metastases. Non-small cell and small cell lung cancer were the prevailing tumor types (78\%). Forty-nine patients (49\%) had normal blood test results (LabBM score 0 points). Their median survival (23 months) was significantly longer than that of patients with higher LabBM score. In multivariate analysis, LabBM score, performance status and single brain metastasis were associated with significantly better survival. Limited extracranial metastases did not impair prognosis. Patients with LabBM score 0 had a 5-year survival rate of 27\% after surgery $(n=24)$ and $39 \%$ after stereotactic radiotherapy $(n=13)$, respectively $(p=0.3)$. Conclusion: Blood biomarkers can be regarded as surrogate of the metastatic burden in the body, which is not always detectable by imaging methods. In contrast to circulating tumor cells and other emerging
\end{abstract}

This article is freely accessible online.

Correspondence to: Dr. Carsten Nieder, Department of Oncology and Palliative Medicine, Nordland Hospital, 8092 Bodø, Norway, Tel: +47 75578449, Fax: +47 75534975, e-mail: carsten.nieder@nlsh.no

Key Words: Stereotactic radiotherapy, whole-brain radiotherapy, brain metastases, oligometastases, prognostic factors, lactate dehydrogenase, $\mathrm{C}$-reactive protein. markers, the LabBM score is inexpensive. Patients with LabBM score $>0$ had a 2.8-fold increased risk of death. The score might be helpful in predicting survival improvement provided by ablative local treatment of oligometastases.

The initially controversial concept of an oligometastatic disease state, an in-between scenario of distant spread that is amenable to long-term survival or cure, in contrast to widely disseminated metastases, has gained increasing acceptance and led to more nuanced treatment approaches (1-5). If these lesions involve the brain, surgical resection and/or stereotactic radiotherapy (SRT) might result in effective local control (6-8). In case of true oligometastases (imaging studies did not miss small tumor deposits), long-term survival can be obtained. However, the limit of detection of metastases under different imaging methods still causes trouble, in terms of undetected widespread disease and short progression-free survival after local treatment to those lesions that were visible $(9,10)$. Emerging approaches such as detection of circulating tumor cells may contribute to higher accuracy, but are not widely available yet, especially in resource-limited settings (11-13). Our group has previously suggested that simple, inexpensive biomarkers based on routine blood tests, e.g. serum lactate dehydrogenase $(\mathrm{LDH})$, may play a role in distinguishing true oligometastatic disease, thus complementing radiological assessment (14). A more comprehensive test panel, the already validated LabBM score $(15,16)$, may further improve the staging process. Besides LDH, C-reactive protein (CRP), albumin, hemoglobin and platelets are included. Completely normal tests result in a LabBM score of 0 . Likely, the score reflects the total tumor burden and associated processes such as inflammation and cachexia (15). Based on previous results with LDH only (14), the hypothesis of the present study was that the LabBM score may improve existing prognostic models, which typically include Karnofsky performance status (KPS) and number of brain metastases $(17,18)$. 
Table I. Patient characteristics.

\begin{tabular}{lrr}
\hline Baseline parameter & Number & Percent \\
\hline Non-small cell lung cancer & 67 & 66 \\
Small cell lung cancer & 12 & 12 \\
Breast cancer & 6 & 6 \\
Melanoma & 8 & 8 \\
Colorectal cancer & 5 & 5 \\
Other primary cancers & 3 & 3 \\
Extracranial metastases & 21 & 21 \\
No extracranial metastases & 80 & 79 \\
Female gender & 50 & 50 \\
Male gender & 51 & 50 \\
One brain metastasis & 58 & 57 \\
Two brain metastases & 25 & 25 \\
Three brain metastases & 11 & 11 \\
Four brain metastases & 7 & 7 \\
Time interval from cancer & & \\
to brain metastases diagnosis & & \\
- Synchronous & 44 & 44 \\
- 1-24 months & 46 & 46 \\
- 25-35 months & 5 & 5 \\
$\quad$ - At least 36 months & 6 & 6 \\
Symptomatic brain metastases & 80 & 79 \\
Staging (imaging) detected asymptomatic & 21 & 21 \\
brain metastases & & \\
Primary tumor controlled & 55 & 54 \\
Primary tumor not controlled or not treated yet & 46 & 46 \\
Stereotactic brain radiotherapy & 27 & 27 \\
Whole-brain radiotherapy & 35 & 35 \\
Surgical resection & 37 & 37 \\
Primary systemic therapy & 2 \\
\hline & & \\
\hline
\end{tabular}

\section{Patients and Methods}

Design. A retrospective study of all patients with maximum 5 (then restricted to 4 , see Results) brain metastases managed at Nordland hospital was performed. These patients had a histological diagnosis obtained from their extracranial primary tumors and metastases in the brain parenchyma (no leptomeningeal disease). The treatment strategy was individualized and the multidisciplinary oncology team selected surgery, SRT, whole-brain radiotherapy (WBRT), appropriate systemic treatment and, if necessary, salvage of new or progressive brain metastases. Systemic treatment was also judged on a case-by-case basis by the hospitals' clinical oncologists. Radical treatment of extracranial metastases was individualized too. The patients were treated between January 01, 2007 and December 31, 2020. Brain magnetic resonance imaging (MRI) was performed to diagnose intracranial metastases and to exclude leptomeningeal dissemination. Extracranial staging relied on computed tomography (CT) without routine positron emission tomography (PET). Patients with extracranial metastases to one organ system, e.g., the lungs, were included, provided the total number of metastatic lesions in the brain and extracranially did not exceed five. Metastases to more than one organ such as liver and bones were not allowed. LDH, CRP, albumin, hemoglobin and platelets were assessed at the start of brain treatment or close to this date (in the week before). The
Table II. Distribution of the LabBM (laboratory values in brain metastases) point sum and related survival outcomes.

\begin{tabular}{lrrc}
\hline Point sum & Number & Percent & Median survival in months \\
\hline 0 & 49 & 49 & 23.0 \\
0.5 & 12 & 12 & 13.0 \\
1 & 16 & 16 & 10.5 \\
1.5 & 13 & 13 & 3.2 \\
2 & 6 & 6 & $7.0(2-3$ points combined $)$ \\
2.5 & 4 & 4 & \\
3 & 1 & 1 & \\
\hline
\end{tabular}

LabBM score (15) was calculated as defined by Berghoff et al. (1 point: LDH or CRP above institutional upper limit of normal, 0.5 points: albumin, hemoglobin or platelets below institutional lower limit of normal; point sum 0-3.5).

Statistical analysis. The methods have already been described in our previous study (14). Overall survival (time to death) from the first day of radiotherapy or surgery was calculated employing the Kaplan-Meier method, and different groups were compared using the log-rank test (SPSS 27, IBM Corp., Armonk, NY, USA). Twenty-two patients were censored after a median follow-up of 20 months. A multivariate analysis (Cox forward conditional regression model) was also performed. We defined statistical significance as $p<0.05$ for all analyses.

\section{Results}

Initial analysis regarding exclusion criteria. We identified 105 patients from the Institution's prospectively maintained database. Due to uncertainty in the best definition of oligometastases (often 5, sometimes 3 or 4 lesions) we looked at the survival stratified by number of brain metastases. The very small group of patients with 5 brain metastases $(n=4)$ had very distinct survival (median only 3.1 months) and was, therefore, excluded. The presence of limited extracranial metastases did not impact on survival. Twenty-one patients had such metastases (lung, skin, adrenal gland, lymph node; radically treated or not) and their median survival was 10.9 months, compared to 12 months in patients with brain-only disease, $p=0.82$.

Final analysis of 101 patients with 1-4 brain metastases. The median age was 65 years, minimum 42 and maximum 90 years. The median KPS was 80, range 50-100. Sixty-seven patients $(66 \%)$ had non-small cell lung cancer (NSCLC). Further patient characteristics are shown in Table I. Patients with single brain metastasis survived longer than those with 2-4 lesions (median 13.3 versus 10.5 months, $p=0.006$ ). The continuous variables age and KPS were also associated with survival ( $p=0.009$ and $p<0.001$, respectively, in univariate 


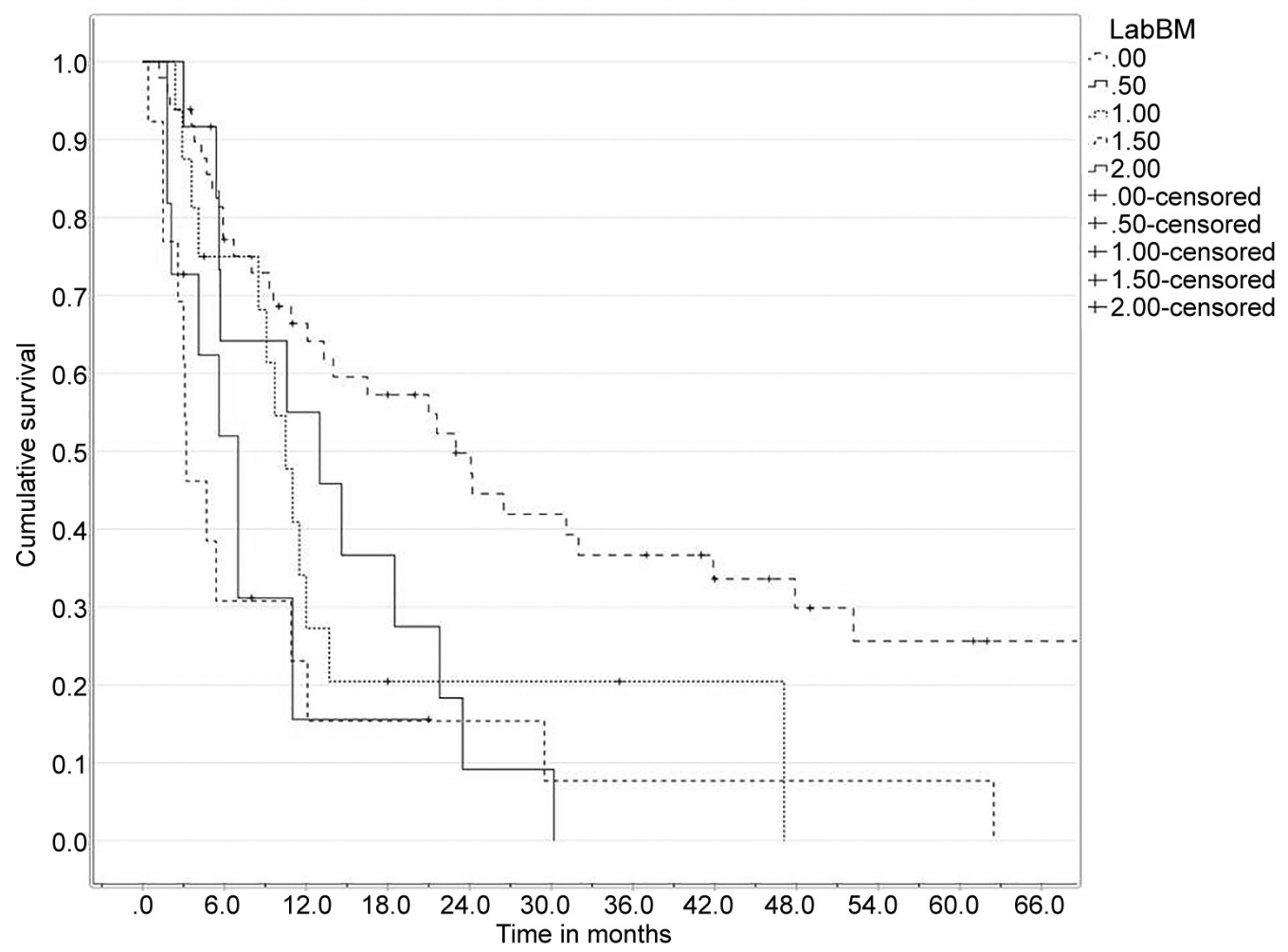

Figure 1. Actuarial overall survival stratified by LabBM score, log-rank test, $p=0.001$.

Cox regression analysis). The other baseline parameters shown in Table I were not significantly associated with actuarial overall survival. Trends towards better survival were observed for time interval $>35$ months and controlled primary tumor $(p=0.1)$.

The proportion of patients with LabBM score 0 was similar for those with 1, 2 and 3 brain metastases (45-52\%) and numerically lower (29\%) if 4 brain metastases were present $(p=0.2)$. A LabBM score of 0 was observed in $46 \%$ of patients with brain-only disease and $57 \%$ of those with additional limited extracranial metastases $(p=0.05$, chi-square test). The score predicted survival outcomes in univariate analysis, Table II and Figure 1. A clearly distinct survival curve was seen in patients with LabBM score 0 . The multivariate Cox regression model included KPS (continuous variable, $p<0.001$ ), age (continuous variable, not significant), single brain metastasis (dichotomized single versus 2-4, $p=0.006$, hazard ratio 0.53 ) and LabBM score (dichotomized 0 versus $0.5-3, p<0.001$, hazard ratio 2.8 ). This analysis confirmed that the score provides important prognostic information. Patients with LabBM score 0 had a 5-year survival rate of $27 \%$ after surgery $(\mathrm{n}=24)$ and $39 \%$ after SRT $(\mathrm{n}=13)$, respectively $(p=0.3)$.

\section{Discussion}

We conducted this study as an expansion of our previous work, which suggested that serum LDH may be a promising biomarker in patients with oligometastases (14), by integrating a larger panel of blood tests (the LabBM score) and enlarging the study population, thereby increasing the statistical power. The underlying hypothesis was that wellknown limitations of imaging-based definition of oligometastases (visible lesions may represent only the tip of the iceberg) can be overcome by assessing other parameters that are surrogates of the true or total burden of disease in the body. As previously discussed by the group which developed the LabBM score (15), patients with widespread metastatic disease are more likely to have a higher score due to inflammation and/or cachexia. Therefore, the survival of patients with true oligometastases (correct radiological staging, no micrometastases, normal blood test results) is expected to be increased compared to those with deceptive oligometastases (additional disease is present and depicted by the additional biomarkers).

Our retrospective study included consecutive patients with brain oligometastases (less than five) and a maximum burden 
of five metastases in total. Some patients had lesions in one extracranial organ system, e.g., bilateral adrenal gland metastases or well localized skin metastases. The majority $(79 \%)$ had brain-only metastases. We found that the number of brain metastases, KPS and LabBM score predicted overall survival. A clear distinction was seen between the KaplanMeier curves of patients with LabBM score 0 (all 5 blood tests were normal) and $\geq 0.5$. The actuarial 5-year survival rate of $26 \%$ (Figure 1) emphasizes that patients with LabBM score 0 are appropriate candidates for aggressive or ablative treatments of all sites of disease. Survival beyond 2 years was also observed in patients with LabBM score $\geq 0.5$. Tailored multidisciplinary treatment approaches should, therefore, be assessed in these patients too. Comparable to a previous analysis (19), we found that brain oligometastases treated at least 3 years after cancer detection were associated with better survival (median 23.5 months, unfortunately only 6 patients belonged to this subset, precluding definitive conclusions).

The present study is limited by the statistical power of subgroup analyses with often $<20$ patients and its retrospective, monocentric design. The diagnosis of an oligometastatic disease extent was not strictly based on PET staging in all patients. Even if employing PET staging, falsenegative and otherwise inaccurate findings cannot be ruled out $(9,10)$. Inexpensive biomarkers that can be obtained in clinical routine may complement imaging-based staging, allowing for better tailored treatment, e.g. by excluding patients with pseudo-oligometastatic malignancies. Hopefully, the rates of overtreatment can be reduced with improved staging accuracy. Emerging biomarkers accessible by liquid biopsy such as circulating tumor cells represent promising tools for optimized decision making and patient selection (11$13,20)$, but are not widely available yet and may generate higher cost than the LabBM score, which can easily be adopted in clinical routine. Automated calculation of the score in electronic patient records appears feasible. In principle, studies have shown that cell-free DNA (cfDNA) in patients with oligometastatic cancer who underwent ablative irradiation for metastatic lesions may provide added value regarding progression-free survival (13). In this example, cfDNA was purified from the plasma of pretreated patients and gene mutations were analyzed by next-generation sequencing.

As repeatedly demonstrated by several authors, radical or ablative local treatment of oligometastatic lesions appears to extend overall survival (21-24). In line with these results, the present study identified long-term survivors in several subgroups despite the absence of mandatory, upfront aggressive local treatment. Some patients managed with initial WBRT were later salvaged with SRT. Our data suggest that the LabBM score may add value when preparing protocols for additional clinical trials.

\section{Conclusion}

Oligometastatic cancer is not always correctly diagnosed, because all available radiological examinations share limitations in terms of certain thresholds for detection of very small metastases. Blood biomarkers reflecting the extent of metastatic cancer appear promising. Patients with LabBM score $>0$ had a 2.8 -fold increased risk of death. The score should be assessed in additional databases from larger Institutions.

\section{Conflicts of Interest}

The Authors declare that they have no competing interests.

\section{Authors' Contributions}

$\mathrm{CN}$ was responsible for the design of the study and performed the statistical analysis. $\mathrm{CN}, \mathrm{AD}$ and $\mathrm{BM}$ conceived the study and drafted the article. All Authors read and approved the final article.

\section{References}

1 Tosoian JJ, Gorin MA, Ross AE, Pienta KJ, Tran PT and Schaeffer EM: Oligometastatic prostate cancer: definitions, clinical outcomes, and treatment considerations. Nat Rev Urol 14(1): 1525, 2017. PMID: 27725639. DOI: 10.1038/nrurol.2016.175

2 Nieder C, Tollåli T, Reigstad A, Pawinski A, Haukland E and Dalhaug A: Oligometastatic non-small cell lung cancer: a significant entity outside of specialized cancer centers? Med Princ Pract 23(6): 526-531, 2014. PMID: 25196201. DOI: $10.1159 / 000365634$

3 Ashworth AB, Senan S, Palma DA, Riquet M, Ahn YC, Ricardi U, Congedo MT, Gomez DR, Wright GM, Melloni G, Milano MT, Sole CV, De Pas TM, Carter DL, Warner AJ and Rodrigues GB: An individual patient data metaanalysis of outcomes and prognostic factors after treatment of oligometastatic non-smallcell lung cancer. Clin Lung Cancer 15(5): 346-355, 2014. PMID: 24894943. DOI: 10.1016/j.cllc.2014.04.003

4 Kobayashi N, Abe T, Noda SE, Kumazaki YU, Hirai R, Igari M, Aoshika T, Saito S, Ryuno Y and Kato S: Stereotactic body radiotherapy for pulmonary oligometastasis from colorectal cancer. In Vivo 34(5): 2991-2996, 2020. PMID: 32871842. DOI: 10.21873 /invivo. 12130

5 Chen H, Louie AV, Higginson DS, Palma DA, Colaco R and Sahgal A: Stereotactic radiosurgery and stereotactic body radiotherapy in the management of oligometastatic disease. Clin Oncol (R Coll Radiol) 32(11): 713-727, 2020. PMID: 32718762. DOI: $10.1016 /$ j.clon.2020.06.018

6 Ampil F, Ellika S, Nanda A and Vora M: Long-term survival after stereotactic radiosurgery of brain metastases: a case series with 10-year follow-up. Anticancer Res 37(9): 5113-5115, 2017. PMID: 28870942. DOI: 10.21873/anticanres.11930

7 Rades D, Blanck O, Khoa MT, VAN Thai P, Hung NQ, Dziggel $\mathrm{L}$ and Schild SE: Validation of a survival score for patients receiving radiosurgery or fractionated stereotactic radiotherapy for 1 to 3 brain metastases. In Vivo 32(2): 381-384, 2018. PMID: 29475924. DOI: 10.21873/invivo.11249

8 Bilger A, Frenzel F, Oehlke O, Wiehle R, Milanovic D, Prokic V, Nieder C and Grosu AL: Local control and overall survival 
after frameless radiosurgery: A single center experience. Clin Transl Radiat Oncol 7: 55-61, 2017. PMID: 29594230. DOI: 10.1016/j.ctro.2017.09.007

9 Lecouvet FE, Oprea-Lager DE, Liu Y, Ost P, Bidaut L, Collette L, Deroose CM, Goffin K, Herrmann K, Hoekstra OS, Kramer G, Lievens Y, Lopci E, Pasquier D, Petersen LJ, Talbot JN, Zacho H, Tombal B and deSouza NM: Use of modern imaging methods to facilitate trials of metastasis-directed therapy for oligometastatic disease in prostate cancer: a consensus recommendation from the EORTC Imaging Group. Lancet Oncol 19(10): e534-e545, 2018. PMID: 30303127. DOI: 10.1016/S1470-2045(18)30571-0

10 deSouza NM, Liu Y, Chiti A, Oprea-Lager D, Gebhart G, Van Beers BE, Herrmann $\mathrm{K}$ and Lecouvet FE: Strategies and technical challenges for imaging oligometastatic disease: Recommendations from the European Organisation for Research and Treatment of Cancer imaging group. Eur J Cancer 91: 153163, 2018. PMID: 29331524. DOI: 10.1016/j.ejca.2017.12.012

11 Yang C, Shi D, Wang S, Wei C, Zhang C and Xiong B: Prognostic value of pre- and post-operative circulating tumor cells detection in colorectal cancer patients treated with curative resection: a prospective cohort study based on ISET device. Cancer Manag Res 10: 4135-4144, 2018. PMID: 30323669. DOI: $10.2147 / C M A R . S 176575$

12 Tinhofer I and Staudte S: Circulating tumor cells as biomarkers in head and neck cancer: recent advances and future outlook. Expert Rev Mol Diagn 18(10): 897-906, 2018. PMID: 30199647. DOI: 10.1080/14737159.2018.1522251

13 Nakamura M, Kageyama SI, Seki M, Suzuki A, Okumura M, Hojo H, Motegi A and Akimoto T: Liquid biopsy cell-free DNA biomarkers in patients with oligometastatic colorectal cancer treated by ablative radiotherapy. Anticancer Res 41(2): 829-834, 2021. PMID: 33517288. DOI: 10.21873/anticanres.14835

14 Nieder C, Dalhaug A and Pawinski A: Serum lactate dehydrogenase contributes to prognostic assessment in patients with oligometastatic cancer and brain involvement. In Vivo 33(1): 229-232, 2019. PMID: 30587628. DOI: 10.21873/invivo.11464

15 Berghoff AS, Wolpert F, Holland-Letz T, Koller R, Widhalm G, Gatterbauer B, Dieckmann K, Birner P, Bartsch R, Zielinski CC, Weller $\mathrm{M}$ and Preusser M: Combining standard clinical blood values for improving survival prediction in patients with newly diagnosed brain metastases-development and validation of the LabBM score. Neuro Oncol 19(9): 1255-1262, 2017. PMID: 28096493. DOI: $10.1093 /$ neuonc/now290

16 Nieder C, Dalhaug A and Pawinski A: External validation of the LabBM score in patients with brain metastases. J Clin Med Res 11(5): 321-325, 2019. PMID: 31019625. DOI: 10.14740/ jocmr 3746

17 Nieder C, Mehta MP, Guckenberger M, Gaspar LE, Rusthoven CG, Sahgal A, Grosu AL and De Ruysscher D: Assessment of extracranial metastatic disease in patients with brain metastases: How much effort is needed in the context of evolving survival prediction models? Radiother Oncol 159: 17-20, 2021. PMID: 33675870. DOI: 10.1016/j.radonc.2021.02.038
18 Nieder C, Mehta MP, Geinitz H and Grosu AL: Prognostic and predictive factors in patients with brain metastases from solid tumors: A review of published nomograms. Crit Rev Oncol Hematol 126: 13-18, 2018. PMID: 29759555. DOI: 10.1016/ j.critrevonc.2018.03.018

19 Nieder C, Mannsåker B and Yobuta R: Late brain oligometastases diagnosed at least 36 months after cancer detection are associated with favorable survival outcome. Cureus 12(1): e6553, 2020. PMID: 32042526. DOI: 10.7759/ cureus. 6553

20 Barnum KJ and Weiss SA: Prognostic and predictive biomarkers in oligometastatic disease. Cancer J 26(2): 100-107, 2020. PMID: 32205533. DOI: 10.1097/PPO.0000000000000438

21 Gomez DR, Blumenschein GR Jr, Lee JJ, Hernandez M, Ye R, Camidge DR, Doebele RC, Skoulidis F, Gaspar LE, Gibbons DL, Karam JA, Kavanagh BD, Tang C, Komaki R, Louie AV, Palma DA, Tsao AS, Sepesi B, William WN, Zhang J, Shi Q, Wang XS, Swisher SG and Heymach JV: Local consolidative therapy versus maintenance therapy or observation for patients with oligometastatic non-small-cell lung cancer without progression after first-line systemic therapy: a multicentre, randomised, controlled, phase 2 study. Lancet Oncol 17(12): 1672-1682, 2016. PMID: 27789196. DOI: 10.1016/S14702045(16)30532-0

22 Pasqualetti F, Montrone S, Vivaldi C, Zani M, Fedele D, Fornaro L, Pasqualetti G, Salvatore L, Manfredi B, Laliscia C, Coraggio G, Gonnelli A, Loupakis F, Masi G, Sainato A, Monzani F, Falcone A and Paiar F: Stereotactic body radiotherapy in patients with lung oligometastases from colorectal cancer. Anticancer Res 37(1): 315-319, 2017. PMID: 28011508. DOI: 10.21873/ anticanres. 11323

23 Stenman M, Sinclair G, Paavola P, Wersäll P, Harmenberg U and Lindskog M: Overall survival after stereotactic radiotherapy or surgical metastasectomy in oligometastatic renal cell carcinoma patients treated at two Swedish centres 2005-2014. Radiother Oncol 127(3): 501-506, 2018. PMID: 29754859. DOI: 10.1016/ j.radonc.2018.04.028

24 Palma DA, Olson R, Harrow S, Gaede S, Louie AV, Haasbeek C, Mulroy L, Lock M, Rodrigues GB, Yaremko BP, Schellenberg D, Ahmad B, Senthi S, Swaminath A, Kopek N, Liu M, Moore K, Currie S, Schlijper R, Bauman GS, Laba J, Qu $\mathrm{XM}$, Warner A and Senan S: Stereotactic ablative radiotherapy for the comprehensive treatment of oligometastatic cancers: long-term results of the SABR-COMET phase II randomized trial. J Clin Oncol 38(25): 2830-2838, 2020. PMID: 32484754. DOI: $10.1200 / J C O .20 .00818$
Received November 9, 2021

Revised November 30, 2021

Accepted December 14, 2021 